\title{
THE USE OF SYNCHROTRON RADIATION IN THE ENERGY CALIBRATION OF ASTRONOMICAL APPARATUS
}

\author{
G. A. GURZADYAN and J.B. OHANESYAN \\ Space Research Branch of Byurakan Astrophysical Observatory, \\ Erevan, Armenian S.S.R., U.S.S.R.
}

The problem of energy calibration of astrophysical apparatus is essential for every or almost every space experiment. The utilization of synchrotron radiation from an electronic accelerator should perhaps be taken as an ideal solution of this problem, if of course, such a possibility is available.

Special equipment for the extraction of synchrotron radiation has been devised at the circular electron accelerator with a maximum electron energy of $6 \mathrm{GeV}$, in the Physics Institute of Erevan (Gurzadyan and Ohanesyan, 1972). The equipment is designed primarily for the energy calibration of astronomical apparatus operating in a vacuum and hard ultraviolet and $X$ rays. However, the equipment can also be applied to a wide range of experiments relating to the physics of solids, crystallography, physics of thin films, $X$ rays, etc.

At present the calibration of astronomical apparatus is carried out by the above synchrotron equipment on a relative energy scale, although the absolute energy calibration of various devices and radiation receivers in all spectral regions - from the optical to the $\mathrm{X}$ rays - is envisaged in the future.

A brief description and basic parameters of the synchrotron equipment are given in the present paper, in addition to preliminary results on the relative energy calibration of astronomical apparatus (for details see Gurzadyan and Ohanesyan, 1971).

The curves of energy distribution in the spectrum of synchrotron radiation from the Erevan accelerator are plotted in Figures 1 and 2 (arbitrary units), showing the wavelength ranges $1-10 \AA$ and $1500-5000 \AA$ for the various values of the maximum energy of electrons. The radiation maxima are all shorter than $10 \AA$; for instance, the maximum is at $0.32 \AA$ when $E=6 \mathrm{GeV}$. The comparatively rapid drop in radiation intensity on the long wavelength side of the maximum assists our plans to use the synchrotron radiation as a monochromatic source in the X-ray region. In this case the radiation maximum can be at any wavelength, by varying the maximum energy of electrons.

The very small dependence of the radiation spectrum on the energy of the electrons in the range above $1500 \AA$ is a characteristic and in practice quite convenient property of synchrotron radiation (Figure 2). Furthermore, such distribution can be adequately expressed by the law $J_{\lambda} \sim \lambda^{-7 / 3}$ for all energies of the electron.

Our synchrotron equipment consists of a steel pipe of gradually increasing diameter from $40 \mathrm{~mm}$ where the accelerator branches off up to $530 \mathrm{~mm}$ at the vacuum-chamber. The overall length of the pipe is $50 \mathrm{~m}$. The angular sizes of the rectangular synchrotron beam are $28^{\prime}$ in the plane of electron motion and $4^{\prime}$ in the perpendicular direction. For 


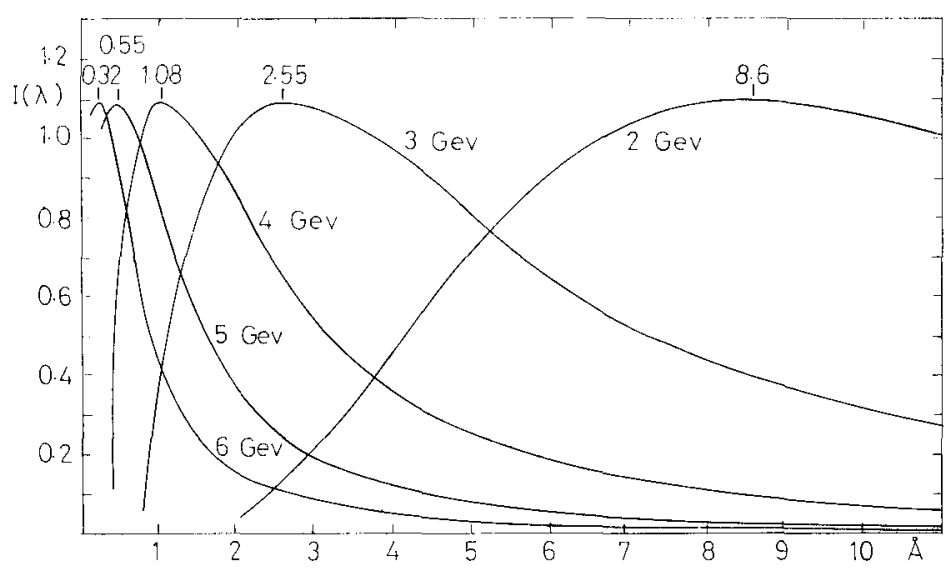

Fig. 1. The distribution of energy in the spectrum of the synchrotron radiation of the Erevan electron accelerator on the wavelength region $1-10 \AA$.

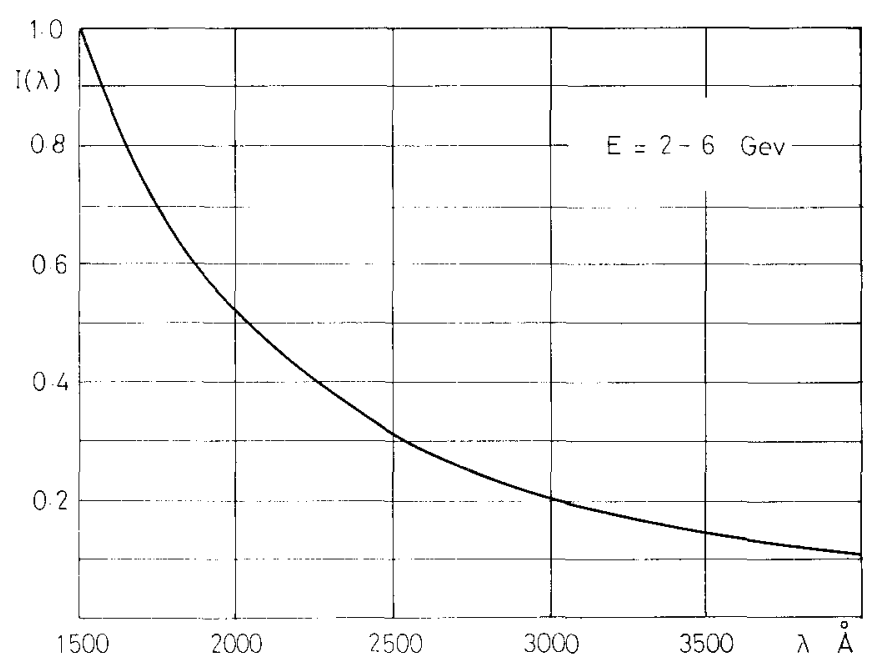

Fig. 2. The same as in Figure 1, but for the region 1500-4000 ̊̊.

these geometrical parameters only $1.3 \times 10^{-3}$ of the total number of photons generated in the accelerator, gets to the vacuum-chamber. The total number of photons obtained from the synchrotron equipment is $4.3 \times 10^{12}$ photons s$^{-1} \AA^{-1}$ at $2000 \AA$, when $E=4 \mathrm{GeV}$. The cross-section of the beam of synchrotron radiation in the vacuumchamber equals nearly $200 \mathrm{~cm}^{2}\left(5\right.$ by $40 \mathrm{~cm}$ ). Hence we have $2.6 \times 10^{10}$ photons $\mathrm{cm}^{-2} \mathrm{~s}^{-1} \AA^{-1}$ for the mean intensity. The intensity of the beam can be reduced by reducing the maximum energy of the electrons, bearing in mind that the intensity of the synchrotron radiation at a given wavelength is proportional to the seventh power of the electron energy $E\left(J_{\lambda} \sim E^{7}\right)$. The intensity of radiation may also be reduced by diaphragming the objective or inlet of the apparatus tested. 
Of major importance is the problem of constancy of the intensity of the synchrotron radiation in time during the calibration operation. Analysis shows that of the two factors influencing the constancy of radiation intensity, viz. the variation in the maximum energy of electrons, and the total number of primary electrons entering from the injector into the circular path of the accelerator, - the second is of greater consequence. As a matter of fact the accuracy of absolute measurements is determined by the stability of the operation of the injector. Special measures are being taken now to ensure the high stability of the operation of the injector. It should be noted, however, that instability of the operation of the injector and accelerator is of no importance at all when a relative energy calibration is carried out, at least in the range longer than $1000 \AA$ A.

The problem of the absolute energy calibration of the apparatus can be solved taking account of the fact that the law of the energy distribution from the $X$ rays through the ultraviolet to the visible region is well known. It is sufficeint to know the absolute intensity of synchrotron photons at any point in this broad region, for example in the visual, by putting a simple photon receiver or electrophotometer just behind the glass window of the vacuum-chamber.

Figure 3 shows examples of spectrograms of synchrotron radiation; they are recorded by means of a short-wave Wadsworth spectrograph, with the Mersenn telescope system as its feeding optics. The spectrograph is designed to obtain slitless spectro-

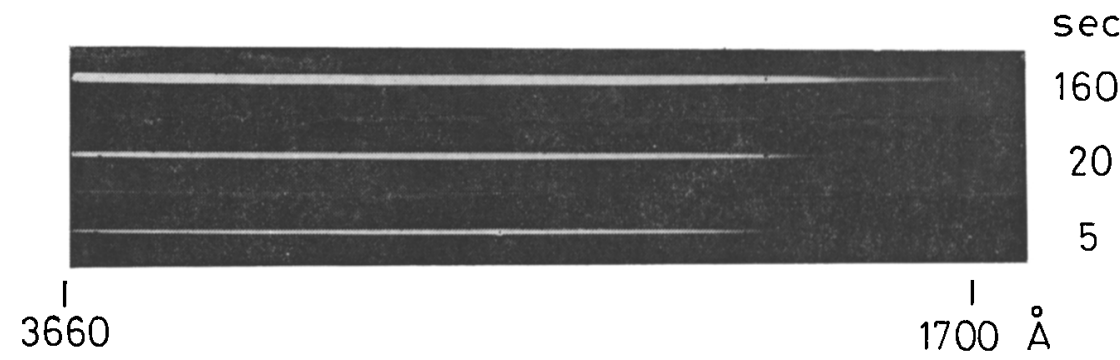

Fig. 3. Examples of spectrograms of synchrotron radiation recorded by the short-wave stellar slitless spectrograph 'Orion'.

grams of the hot stars from above the atmosphere in the wave-length range 2000 $3800 \AA$. The optical scheme of the telescope-spectrograph is given in Gurzadyan and Ohanesyan (1972). It consists of the following elements:

- Two parabolic mirrors coated with aluminium;

- One concave blazed diffraction grating with 1200 lines per $\mathrm{mm}$, ruled in aluminum (the curvature radius is $500 \mathrm{~mm}$ and the dimensions of ruled portion are $50 \times 55 \mathrm{~mm}$ );

- One parallel-sided quartz plate $2 \mathrm{~mm}$ thick (for cutting off the second order of the spectrograph);

- A film of the UFSH-4 type (Kalinkina et al., 1965), as a detector.

The angle between the optical axis of the telescope and the spectrograph is 18.5 . 
The spectrograms are made through the inlet diaphragm, $2 \times 2 \mathrm{~mm}$ in size and are exposed from several seconds to minutes. As a result of the development of these spectrograms and their comparision with the known distribution of the energy in the spectrum of the synchrotron radiation (Figure 2), the so-called reduction curve $\delta_{\lambda}$ of the above telescope-spectrograph has been found, this being our final aim. The curve is plotted in Figure 4, where $\delta_{\lambda}$ is taken as the unity on the wavelength $2500 \mathrm{~mm}$. This curve is given without correction for the astigmatism of our optical system.

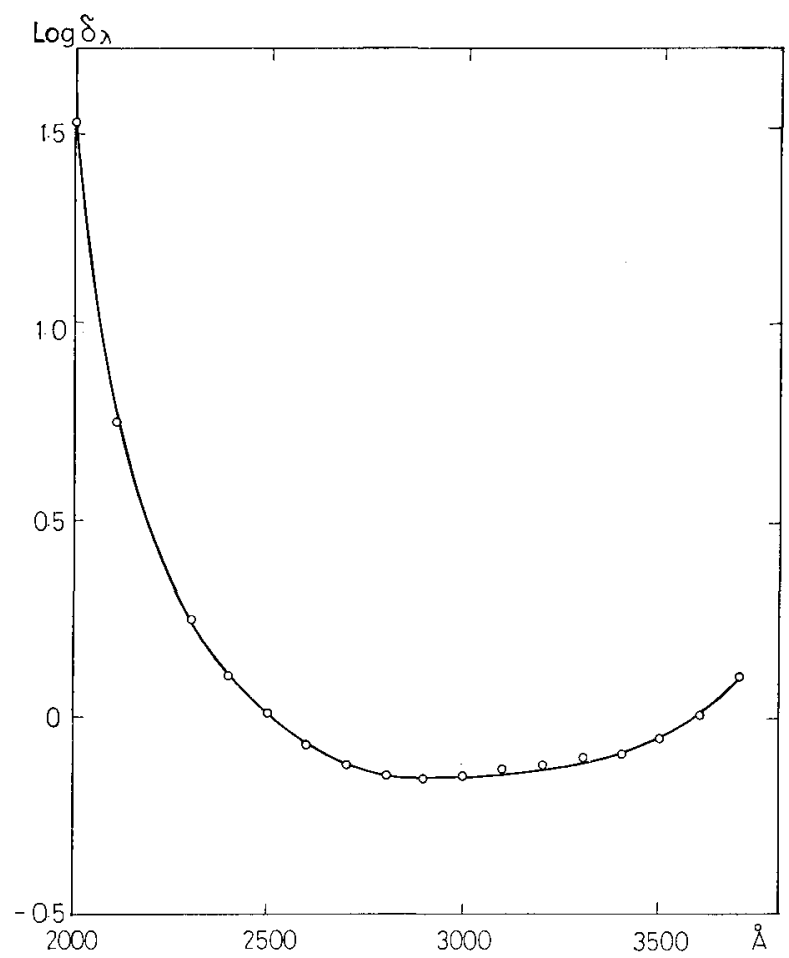

Fig. 4. The reduction curve for the short-wave stellar spectrograph 'Orion'.

With the reduction curve available, it is not difficult to find out the true distribution of the energy (on a relative scale) in the continous spectrum of any star, the spectrogram of which has been obtained with the help of the above spectrograph. It is only necessary to multiply the intensity of the continuous spectrum of the star by the corresponding dimensionless quantity $\delta_{\lambda}$, taken from Figure 4 . The errors in the given reduction curve do not exceed $\pm 10 \%$ in the range of wavelengths $3400-2400 \AA$; above $3400 \AA$ and below $2400 \AA$ the errors are somewhat larger.

It is necessary to add in conclusion that some difficulties are presented in locating the apparatus tested in relation to the synchrotron beam, which has a strongly directional character. 


\section{References}

Gurzadyan, G. A. and Ohanesyan, J. B.: 1971, Soviet Astron. AJ, 48, 1289.

Kalinkina, T. A., Oshurkova, A. N., Pankova, A. A., Uvarova, V. M., Tshistova, G. I., and Shpolskij, M. R.: 1965, J. Priclad. Spectr. 2, 475.

Gurzadyan, G. A. and Ohanesyan, J. B.: 1972, this issue, p. 647. 